\title{
An Analysis of Monotone Follower Problems for Diffusion Processes ${ }^{* \dagger}$
}

\author{
Erhan Bayraktar Masahiko Egami $^{\ddagger}$
}

\begin{abstract}
We consider a singular stochastic control problem, which is called the Monotone Follower Stochastic Control Problem and give sufficient conditions for the existence and uniqueness of a local-time type optimal control. To establish this result we use a methodology that has not been employed to solve singular control problems. We first confine ourselves to local time strategies. Then we apply a transformation to the total reward accrued by reflecting the diffusion at a given boundary and show that it is linear in its continuation region. Now, the problem of finding the optimal boundary becomes a non-linear optimization problem: The slope of the linear function and an obstacle function need to be simultaneously maximized. The necessary conditions of optimality come from first order derivative conditions. We show that under some weak assumptions these conditions become sufficient. We also show that the local time strategies are optimal in the class of all monotone increasing controls.

As a byproduct of our analysis, we give sufficient conditions for the value function to be $\mathbf{C}^{2}$ on all its domain. We solve two dividend payment problems to show that our sufficient conditions are satisfied by the examples considered in the mainstream literature. We show that our assumptions are satisfied not only when capital of a company is modeled by a Brownian motion with drift but also when we change the modeling assumptions and use a square root process to model the capital.
\end{abstract}

\section{Introduction}

We solve a class of singular control problems which are known as Monotone Follower Stochastic Control Problems (see Karatzas and Shreve [7] for the terminology) for a general class of diffusion processes. In particular, we give necessary and sufficient conditions under which the continuation region is constituted by a single open interval in the state space of the controlled process. To establish our main result, we first restrict ourselves to local-time strategies, each of which corresponds controlling the underlying diffusion by reflecting it at a particular point. Applying a particular transformation to the total reward accrued by reflecting the diffusion at a given boundary, we show that the transformed reward is linear in its continuation region. The slope is a function of the boundary point. In the rest of the state space, in the region of action, the transformed reward is equal to an obstacle, which also depends on the value of the boundary point. This transforms finding the optimal boundary to a nonlinear optimization problem: The slope and the obstacle have to be maximized simultaneously. We give the necessary conditions of optimality using the first order derivative conditions and show that under some weak

\footnotetext{
* 2000 Mathematics Subject Classification. Primary: 93E20, Secondary:60J60

${ }^{\dagger}$ Key Words. Singular stochastic control, monotone follower problem, one-dimensional diffusions.

${ }^{\ddagger}$ E. Bayraktar is supported in part by the National Science Foundation, under grant DMS-0604491.

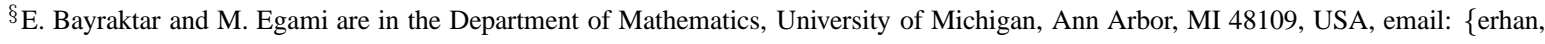
egami\}@umich.edu.
} 
assumptions these conditions become sufficient. That is, our methodology of identifying the unique solution of the singular control problem relies on a combination of the classical diffusion theory, which helps us give a geometric characterization of the value function (the optimal reward), and non-linear programming. Next, we show that the local time strategies are optimal in the class of monotone increasing strategies under some certain assumptions.

Among the benefits of our analysis are the sufficient conditions we provide for the value function to be $\mathbf{C}^{2}$ in the entire state space. This sheds light on the heuristic principle of smooth fit, which suggests that the value function is $\mathbf{C}^{2}$ across the boundary that demarcates the regions of action and inaction. Our approach should be contrasted with the ad hoc ordinary Hamilton-Jacobi-Bellman (HJB) approach, which assumes the principle of smooth fit to construct a solution. There is no guarantee that a solution could be found and using that method it is hard to establish sufficient conditions under which a solution exists. And even if a solution is constructed to the quasi-variational inequalities, one still has to verify whether the solution satisfies the assumptions of a verification lemma, i.e., verify the optimality. For further details of this approach see e.g. Øksendal and Sulem [9].

To illustrate our results we consider the dividend payment problem for two different scenarios. First, we take the cash-flow of a company to be a Brownian motion with drift. (This case was analyzed by Jeanblanc and Shiryaev [6] using the ordinary HJB approach.) Second, we take the cash flow of the company to be a square root process. In this case we show that the optimal reflection level is strictly less than the mean-reversion level. In the second example the functions in terms of which the sufficient conditions are stated are only available in terms some special functions. Yet, we are able to prove that the sufficient conditions in our theorems are satisfied by only analyzing the ordinary differential equation these functions satisfy without making a reference to their explicit representations. This gives us a method to check the sufficient conditions for other diffusions even when explicit representations are not available. We also extend our results to solve constrained optimization problems. A dividend payout problem with solvency constraints was recently solved by Paulsen [10]. In this problem, the firm is allowed to pay dividends only if the cash flow process is greater than certain (pre-determined) value. Here, we provide a simpler proof to Paulsen's result, by a very simple modification of the proof of Proposition 2.3, which characterization provided for the optimal reflection barrier.

A similar methodology to ours was used by Dayanik and Karatzas [3], to give a general characterization of the value function of the optimal stopping problem of one dimensional diffusions. The value function of the optimal stopping problem (up to a transformation) is characterized as a concave majorant of a fixed obstacle. In the singular control problem we analyze, the obstacle is not fixed. When we apply the same transformation to the reward corresponding to the control that is identified by a fixed boundary, the transformed reward becomes linear in the region of inaction whose slope depends on this boundary point. On the region of action the transformed reward is an obstacle and is a function of the boundary point. Therefore, we maximize the slope of the linear function and the obstacle simultaneously over all possible boundary points to obtain the optimal boundary. As a result, we characterize the optimal boundary first and compute the value function (the optimal reward) given this characterization, whereas [3] characterize the value function first and then compute the optimal boundary using this characterization.

Dayanik and Egami [4], Bayraktar and Egami [1] (in this work effects of implementation delay are taken into account) also use similar methodologies. However, the results we obtained here can not be obtained from the results of these papers. In these papers, we could not characterize the optimal control policy completely. The boundary of the region of action and inaction can be completely characterized only when the threshold that the state process is taken to, after the application of the control, is given. Therefore, the characterization of the optimal boundary that we obtain here for the singular control problem can not be obtained using a limiting argument (as the fixed cost goes to zero). Also, the two problems are very different in nature. For example, the singular control problem 
is smoother than the impulse control problem. In the impulse control problem, given a particular policy, both the slope of the transformed reward in the region of inaction and the obstacle (the transformed value function in the region of action) can be determined using the fact that it is continuous at the boundary. However, determining the slope of the transformed reward in the singular control problem is trickier. To write down the slope of the transformed reward in the region of inaction as a function of the boundary, we first show that the transformed reward is $\mathbf{C}^{1}$. The continuity of the first derivative is also used to determine the obstacle (the transformed reward in the region of action) as a function of the boundary point. On the other hand, the proof of optimality of local time strategies among a more general class of controls in the case of singular control problem differs significantly from the optimality proof of the threshold strategies in the case of impulse control problem. The latter uses the fact that the value function (optimal reward) of the impulse control problem can be approximated by a sequence of optimal stopping problems.

The rest of the paper is organized as follows: In section 2, we solve the monotone follower problem for a general diffusion. We first find the optimal local time strategy. In Section 2.1, we state the problem, in Section 2.2, we characterize the value function corresponding to a given boundary and after applying a particular transformation value function becomes linear in the region of inaction. In Section 2.3, we characterize the optimal local time control. We also extend our analysis to solve a constrained optimization problem. In Section 2.4, we show that the local time strategies are optimal among all admissible monotone controls. Here, we also point out that under the assumptions of Proposition 2.1 the value function is $\mathbf{C}^{2}$. In Section 2.5, we solve the dividend payment problem when the capital of a company is assumed to be either Brownian motion with drift or a square root process. We collect some preliminary results to Section 3 , which is our appendix.

\section{Solution of Monotone Follower Problems}

\subsection{Reflected Diffusions}

Let $\left(\Omega, \mathcal{F},\left(\mathcal{F}_{t}\right)_{t \geq 0}, \mathbb{P}\right)$ be a complete probability space with a standard Brownian motion $W=\left\{W_{t} ; t \geq 0\right\}$ and consider the diffusion process $X^{0}$ with state pace $\mathcal{I}=[c, d) \subseteq \mathbb{R}$ and dynamics

$$
d X_{t}^{0}=\mu\left(X_{t}^{0}\right) d t+\sigma\left(X_{t}^{0}\right) d W_{t}
$$

for some Borel functions $\mu: \mathcal{I} \rightarrow \mathbb{R}$ and $\sigma: \mathcal{I} \rightarrow(0, \infty)$. (We assume that the functions $\mu$ and $\sigma$ are sufficiently regular so that (2.1) makes sense.) We use " 0 " as the superscript to indicate that $X^{0}$ is uncontrolled. We denote the infinitesimal generator of $X^{0}$ by $\mathcal{A}$ and consider the $\operatorname{ODE}(\mathcal{A}-\alpha) v(x)=0$. This equation has two fundamental solutions, $\psi(\cdot)$ and $\varphi(\cdot)$. We set $\psi(\cdot)$ to be the increasing and $\varphi(\cdot)$ to be the decreasing solution. 1 We will take $c$ to be absorbing and $d$ to be natural, and therefore $\psi(d-)=\infty, \varphi(d-)=0$ since $X^{0}$ never reaches $d$. First, we define an increasing function

$$
F(x) \triangleq \frac{\psi(x)}{\varphi(x)} .
$$

\footnotetext{
${ }^{1}$ In fact, defining $\tau_{r}^{0} \triangleq \inf \left\{t \geq 0: X_{t}^{0}=r\right\}$, for every $r \in(c, d)$, we can write

$$
\psi(x)=\left\{\begin{array}{ll}
\mathbb{E}^{x}\left[e^{\left.-\alpha \tau_{y}^{0}\right],},\right. & \text { if } x \leq y \\
1 / \mathbb{E}^{y}\left[e^{-\alpha \tau_{x}^{0}}\right], & \text { if } x>y
\end{array}, \quad \varphi(x)=\left\{\begin{array}{ll}
1 / \mathbb{E}^{y}\left[e^{-\alpha \tau_{x}^{0}}\right], & \text { if } x \leq y, \\
\mathbb{E}^{x}\left[e^{-\alpha \tau_{y}^{0}}\right], & \text { if } x>y
\end{array},\right.\right.
$$
}

for every $x \in \mathcal{I}$ and an arbitrary but fixed $y \in \mathcal{I}$ (see Itô and McKean [5]). 
Next, we define concavity of a function with respect $F$ as follows: A real valued function $u$ is called $F$-concave on $(c, d)$ if, for every $c \leq l<r \leq d$ and $x \in[l, r]$,

$$
u(x) \geq u(l) \frac{F(r)-F(x)}{F(r)-F(l)}+u(r) \frac{F(x)-F(l)}{F(r)-F(l)} .
$$

Consider the solution of $(X, Z)$ of the stochastic differential equation with reflection

$$
d X_{t}=\mu\left(X_{t}\right) d t+\sigma\left(X_{t}\right) d W_{t}-d Z_{t}, \quad X_{0-}=x \in(c, d),
$$

where $Z=\left(Z_{t}\right)_{t \geq 0}$ is a continuous non-decreasing (except at $\left.t=0\right)\left\{\mathcal{F}_{t}\right\}$-adapted process such that

$$
Z_{t}-Z_{0}=\int_{(0, t)} 1_{\left\{X_{s}=b\right\}} d Z_{s}
$$

for some $b \in(c, d)$. Here, we use the same notation as [6], see equations (4.7) and (4.8). To emphasize the fact that the initial value of the process $Z, Z_{0}$, depends on $X_{0-}=x$, below we denote it by $Z_{0}(x)$. We assume that $x \rightarrow Z_{0}(x), x \in(c, d)$, is a measurable function and $Z_{0}(c)=0$.

Here, $Z$ is called the local time of the process $X$ at point $b$. When this control is applied to the state process $\left\{X_{t}\right\}_{t \geq 0}$, for $t>0$, it moves in $(c, b]$ and it is reflected at $b$ (until the time of absorption). First, we will find the best local time strategy. We will denote the set of local time strategies by $\mathfrak{A}$. Next, in Proposition 2.3, we will show that under some certain assumptions the local time strategies are optimal in a larger class of controls, namely non-decreasing, $\left\{\mathcal{F}_{t}\right\}$-adapted controls. Let $\tau_{c} \triangleq \inf \left\{t \geq 0: X_{t}=c\right\}$. We consider the following performance measure associated with $Z \in \mathfrak{A}$

$$
J^{Z}(x)=h \cdot Z_{0}(x)+\mathbb{E}^{x-Z_{0}(x)}\left[\int_{0}^{\tau_{c}} e^{-\alpha s} f\left(X_{s}\right) d s+h \int_{\left(0, \tau_{c}\right)} e^{-\alpha s} d Z_{s}\right],
$$

for some given $h \in \mathbb{R}_{+}$. Here, $\mathbb{P}^{x-Z_{0}(x)}\{\cdot\}$ is a short-hand notation for the conditional probability measure $\mathbb{P}\left\{\cdot \mid X_{0}=x-Z_{0}(x)\right\}$ and $\mathbb{E}^{x-Z_{0}(x)}$ is the expectation with respect to that probability measure. In (2.6), we used the following notation

$$
h \int_{\left(0, \tau_{c}\right)} e^{-\alpha s} d Z_{s} \triangleq h \int_{0}^{\tau_{c}} e^{-\alpha s} d Z_{s}-h Z_{0}(x)
$$

The objective is to find the optimal strategy $Z^{*} \in \mathfrak{A}$ (if it exists) and the value function:

$$
v(x) \triangleq \sup _{Z \in \mathfrak{A}} J^{Z}(x)=J^{Z^{*}}(x) .
$$

One could choose $\mathfrak{A}$ to be the family of non-decreasing, $\left\{\mathcal{F}_{t}\right\}_{t \geq 0}$-adapted process. We will show in Proposition2.3 that it is enough to consider only the local time strategies under certain assumptions.

Assumption 2.1. The function $f:(c, d) \rightarrow \mathbb{R}$ is continuous and satisfies

$$
\mathbb{E}^{x}\left[\int_{0}^{\infty} e^{-\alpha s}\left|f\left(X_{s}^{0}\right)\right| d s\right]<\infty .
$$

\subsection{Characterization of the Value Function Corresponding to a Given Reflection Level}

We will first obtain a dynamic programming equation for the performance measure (2.6). Next, we will apply a transformation to linearize the difference between the value associated with a particular control and the value 
associated with not applying any control at all. Recall that the region in which the particular control prescribes no action is commonly referred to as the continuation region or inaction region of this particular control.

Let $\tau_{b} \triangleq\left\{t \geq 0: X_{t} \geq b\right\}$. On denoting

$$
g(x) \triangleq \mathbb{E}^{x}\left[\int_{0}^{\infty} e^{-\alpha s} f\left(X_{s}^{0}\right) d s\right]
$$

for $x \in[c, b]$, we can write

$$
\begin{aligned}
\mathbb{E}^{x-Z_{0}(x)} & {\left[\int_{0}^{\tau_{c}} e^{-\alpha s} f\left(X_{s}\right) d s\right]=\mathbb{E}^{x}\left[\int_{0}^{\tau_{c}} e^{-\alpha s} f\left(X_{s}\right) d s\right] } \\
& =\mathbb{E}^{x}\left[\int_{0}^{\tau_{b} \wedge \tau_{c}} e^{-\alpha s} f\left(X_{s}^{0}\right) d s+e^{-\alpha\left(\tau_{b} \wedge \tau_{c}\right)} \mathbb{E}^{X_{\tau_{b}} \wedge \tau_{c}} \int_{0}^{\tau_{c}} e^{-\alpha s} f\left(X_{s}\right) d s\right] \\
& =g(x)-\mathbb{E}^{x}\left[e^{-\alpha\left(\tau_{c} \wedge \tau_{b}\right)} g\left(X_{\tau_{b} \wedge \tau_{c}}^{0}\right)\right]+\mathbb{E}^{x}\left[e^{-\alpha\left(\tau_{b} \wedge \tau_{c}\right)} \mathbb{E}^{X_{\tau_{b} \wedge \tau_{c}}} \int_{0}^{\tau_{c}} e^{-\alpha s} f\left(X_{s}\right) d s\right] \\
& =g(x)-\mathbb{E}^{x}\left[e^{-\alpha\left(\tau_{b} \wedge \tau_{c}\right)} g\left(X_{\left(\tau_{b} \wedge \tau_{c}\right)-}\right)\right]+\mathbb{E}^{x}\left[e^{-\alpha\left(\tau_{b} \wedge \tau_{c}\right)} \mathbb{E}^{X_{\tau_{b} \wedge \tau_{c}}} \int_{0}^{\tau_{c}} e^{-\alpha s} f\left(X_{s}\right) d s\right],
\end{aligned}
$$

in which the third line follows from Lemma 3.1 in the Appendix. Therefore, for $x \in(c, b]$

$$
\begin{aligned}
J^{Z}(x) & =\mathbb{E}^{x}\left[e^{-\alpha\left(\tau_{b} \wedge \tau_{c}\right)}\left\{-g\left(X_{\left(\tau_{b} \wedge \tau_{c}\right)-}\right)+\mathbb{E}^{X_{\tau_{b} \wedge \tau_{c}}}\left[\int_{0}^{\tau_{c}} e^{-\alpha s} f\left(X_{s}\right) d s+\int_{\left(0, \tau_{c}\right)} e^{-\alpha s} h d Z_{s}\right]\right\}\right]+g(x) \\
& =\mathbb{E}^{x}\left[e^{-\alpha\left(\tau_{b} \wedge \tau_{c}\right)}\left\{-g\left(X_{\left(\tau_{b} \wedge \tau_{c}\right)-}\right)+J^{Z}\left(X_{\tau_{b} \wedge \tau_{c}}\right)\right\}\right]+g(x) .
\end{aligned}
$$

Let us define

$$
u^{b}(x) \triangleq J^{Z}(x)-g(x), \quad x \in[c, d) .
$$

It is worth noting that $u^{b}(c)=-g(c)$ since $J^{Z}(c)=0$.

Equation (2.10) can be written as

$$
\begin{aligned}
u^{b}(x) & =\mathbb{E}^{x}\left[e^{-\alpha\left(\tau_{b} \wedge \tau_{c}\right)}\left\{u^{b}\left(X_{\tau_{b} \wedge \tau_{c}}\right)-g\left(X_{\left(\tau_{b} \wedge \tau_{c}\right)-}\right)+g\left(X_{\tau_{b} \wedge \tau_{c}}\right)\right\}\right] \\
& =\mathbb{E}^{x}\left[e^{-\alpha\left(\tau_{b} \wedge \tau_{c}\right)} u^{b}\left(X_{\tau_{b} \wedge \tau_{c}}\right)\right]
\end{aligned}
$$

for $x \in(c, b]$. On the other hand, if $x \in[b, d)$, then

$$
u^{b}(x)=h \cdot(x-b)-g(x)+g(b)+u^{b}(b), \quad x \in[b, d) .
$$

Using (2.12) and (2.13) can be written in a more compact form as

$$
u^{b}(x)= \begin{cases}u_{0}^{b}(x) \triangleq \mathbb{E}^{x}\left[1_{\left\{\tau_{b}<\tau_{c}\right\}} e^{-\alpha \tau_{b}} u^{b}(b)+1_{\left\{\tau_{b}>\tau_{c}\right\}} e^{-\alpha \tau_{c}} u^{b}(c)\right], & x \in[c, b], \\ K(x, b)+u_{0}^{b}(b), & x \in[b, d),\end{cases}
$$

in which

$$
K(x, y) \triangleq h \cdot(x-y)-g(x)+g(y) .
$$

Observe that $u^{b}(x)$ is continuous at $x=b$.

Using Lemma3.2 we can write the function $x \rightarrow u_{0}^{b}(x), x \in(c, b]$ as

$$
u_{0}^{b}(x)=u^{b}(b) \frac{\psi(c) \varphi(x)-\psi(x) \varphi(c)}{\psi(c) \varphi(b)-\psi(b) \varphi(c)}+u^{b}(c) \frac{\psi(x) \varphi(b)-\psi(b) \varphi(x)}{\psi(c) \varphi(b)-\psi(b) \varphi(c)} .
$$


The function $x \rightarrow u_{0}^{b}(x), x \in(c, b]$ can be linearized by using

$$
W_{0}^{b}(x) \triangleq\left(u_{0}^{b} / \varphi\right) \circ F^{-1}(x), \quad x \in[F(c), F(b)],
$$

and $(2.16)$ becomes

$$
W_{0}^{b}(x)=W_{0}^{b}(F(c)) \frac{F(b)-x}{F(b)-F(c)}+W_{0}^{b}(F(b)) \frac{x-F(c)}{F(b)-F(c)}, \quad x \in[F(c), F(b)] .
$$

We extend the function $x \rightarrow W_{0}^{b}(x)$, from $x \in[F(c), F(b)]$ to $[F(c), F(d))$ by defining

$$
W^{b}(x) \triangleq\left(u^{b} / \varphi\right) \circ F^{-1}(x), \quad x \in[F(c), F(d)) .
$$

We have now established that $W^{b}(x)$ is a linear function in the transformed continuation region (the region of no action). Note that (2.14) and (2.18) do not completely determine $u^{b}$ : the slope and the intercept of the line $W^{b}(x) x \in[F(c), F(b)]$ need to be determined. But we already know that

$$
\text { the linear function } W_{0}^{b}(\cdot) \text { passes through }\left(F(c), l_{c}\right)=\left(F(c), \frac{-g(c)}{\varphi(c)}\right) .
$$

The slope of this linear function will be determined as a function of $b$, i.e., $b \rightarrow \beta(b), b \in(c, d)$. Then, we will give sufficient conditions in Proposition2.1 under which the optimal $b^{*}$, i.e. $b \in(c, d)$ such that $u^{b^{*}}(x)=v(x)-g(x)$, can be determined by the ordinary first order condition, i.e. as the unique solution of $\partial \beta(b) / \partial b=0$.

\subsection{Characterization of the Optimal Reflection Level}

In this section, we characterize the optimal level at which the diffusion is to be reflected to maximize a given reward functional as the unique solution of a non-linear equation. We first transform the function $K(\cdot)$, defined in (2.15), into

$$
R(x ; b) \triangleq \frac{K\left(F^{-1}(x), b\right)}{\varphi\left(F^{-1}(x)\right)}, \quad x \in[F(b), F(d)) .
$$

From (2.18) and (2.20) it follows that

$$
W^{b}(x)=\beta(x-F(c))+l_{c}, \quad x \in[F(c), F(b)],
$$

for some $\beta \in \mathbb{R}$, which is to be determined as a function of $b$. Our task in this section is to identify an appropriate slope $\beta^{*}=\beta\left(b^{*}\right)$, so that the function $b \rightarrow W^{b}(x)$ is maximized at $b^{*}$ for any $x \in(c, d)$.

Proposition 2.1. Let us define $k: \mathbb{R} \rightarrow \mathbb{R}$ by

$$
k(x) \triangleq h-g^{\prime}(x)-l_{c} \varphi^{\prime}(x) .
$$

Assume that: (i) For any $b \in[c, d), R(y ; b)$ defined in (2.21) is differentiable with respect to $y$; (ii) For any $b \in[c, d), x \rightarrow R(x ; b)+\frac{\varphi(b)}{\varphi\left(F^{-1}(x)\right)} W(F(b))$ is increasing and concave on $x \in(F(j), F(d))$ for some point $j \in(c, d)$ and it approaches infinity as $x \rightarrow d$; (iii) There exists a unique solution $b^{*} \in(c, d)$ to the equation

$$
k^{\prime}(b) \psi^{\prime}(b)-k(b) \psi^{\prime \prime}(b)+F(c)\left[k(b) \varphi^{\prime \prime}(b)-k^{\prime}(b) \varphi^{\prime}(b)\right]=0
$$

such that $b^{*}$ satisfies

$$
k^{\prime \prime}\left(b^{*}\right) \psi^{\prime}\left(b^{*}\right)-k\left(b^{*}\right) \psi^{\prime \prime \prime}\left(b^{*}\right)+F(c)\left(-k^{\prime \prime}(b) \varphi^{\prime}(b)+k(b) \varphi^{\prime \prime}(b)\right)<0 .
$$


Then the solution of (2.7) is given by $v(x)=u^{b^{*}}(x)+g(x)$, in which $u^{b^{*}}$ is given by the equation (2.14) if we replace by by $b^{*}$ and choose the slope of $(2.18)$ to be $\beta^{*}$, which is given by

$$
\beta^{*} \triangleq \frac{k\left(b^{*}\right)}{\psi^{\prime}\left(b^{*}\right)-F(c) \varphi^{\prime}\left(b^{*}\right)} .
$$

Recall that $W^{b^{*}}(x)$ is linear for $x \in\left[F(c), F\left(b^{*}\right)\right)$.

Proof. We will first determine the slope of the line in (2.22), as a function of $b$, i.e., $b \rightarrow \beta(b), b \in[c, d)$. This will be established by showing that $W^{b}$ defined in (2.19) is continuously differentiable at $b$. To this end we will first consider the threshold strategy that is characterized by the pair $(b, a) \in[c, d)^{2}: Z$ is said to be a threshold strategy corresponding to $(b, a)$ if, whenever the process $X$ in (2.4) hits level $a$ or is above $a$, then it jumps to (the jump is forced by $Z$ ) level $b \leq a$. (Although, the letter $Z$ was used to denote only local time strategies before we would like to use it to denote the threshold strategies to be able to refer (2.4) when we are describing threshold strategies. This prevents introducing unnecessary equations.) Consider the reward in 2.6 corresponding to the particular threshold strategy $Z$ and denote it by $u^{b, a}$. Note that $u^{b, b}=u^{b}$. The control represented by the pair $(b, a)$ is of impulse control type. For $b \in[c, d)$, let us find $a(b)$ such that $\sup _{a \in[c, d)} u^{b, a}=u^{b, a(b)}$. Using the results in Section 2.2 of [1] and the assumption (i) and (ii) of the proposition we conclude that, for any $b$, there exists a unique $a(b)$ such that the function $x \rightarrow W^{b, a(b)}(x)$, defined by (2.19) when $u$ is replaced by $u^{b, a(b)}$, is continuously differentiable at $a(b)$. This characterization of the function $b \rightarrow a(b), b \in[c, d)$ will be used to show $a(b)=b, b \in[c, d)$ and to calculate the slope $b \rightarrow \beta(b), b \in[c, d)$.

Let us define

$$
\tilde{W}^{b}(x) \triangleq \begin{cases}\beta(b)(x-F(c))+l_{c} & x \in[F(c), F(b)] \\ H(x, b) \triangleq R(x ; b)+\frac{\varphi(b)}{\varphi\left(F^{-1}(x)\right)}\left(\beta(b)(F(b)-F(c))+l_{c}\right), & x \in[F(b), F(d)) .\end{cases}
$$

in which

$$
\beta(b)=\frac{k(b)}{\psi^{\prime}(b)-F(c) \varphi^{\prime}(b)} .
$$

The right-hand derivative of the function $\tilde{W}^{b}$ satisfies

$$
\begin{aligned}
& \left(\tilde{W}^{b}\right)^{\prime}(F(b)+)=-\left(\beta(b)(F(b)-F(c))+l_{c}\right) \frac{1}{\varphi(b)} \frac{\varphi^{\prime}(b)}{F^{\prime}(b)}+\left.\frac{\partial}{\partial x} R(x ; b)\right|_{x=F(b)} \\
& =-\left(\beta(b)(F(b)-F(c))+l_{c}\right) \frac{1}{\varphi(b)} \frac{\varphi^{\prime}(b)}{F^{\prime}(b)}+\left.\frac{\varphi(x)\left(-g^{\prime}(x)+h\right)-(h \cdot(x-b)-g(x)+g(b)) \varphi^{\prime}(x)}{\varphi(x)^{2}} \frac{1}{F^{\prime}(x)}\right|_{x=b} \\
& =\beta(b),
\end{aligned}
$$

where we used (2.15) and (2.21) to derive the first equality, and (2.28) to derive the third inequality. The function $x \rightarrow \tilde{W}^{b}(x), x \in[F(c), F(d))$ is $C^{1}$ at $x=F(b)$, since the left-hand derivative is also $\beta(b)$. This implies that $\tilde{W}^{b}=W^{b, a(b)}$ or $\left(u^{b, a(b)} \varphi\right) \circ F^{-1}=\tilde{W}^{b}$ and that $a(b)=b$. As a result we see that $W^{b}$ satisfies smooth fit condition at $b$ and the slope in (2.22) is given by (2.28). Before we continue with the proof the reader should note that

$$
\begin{aligned}
\beta(b) & =\lim _{a \downarrow b} \frac{R(F(a) ; b))+l_{c}\left(\frac{\varphi(b)}{\varphi(a)}-1\right)}{F(a)-\frac{\varphi(b)}{\varphi(a)} F(b)+F(c)\left(-1+\frac{\varphi(b)}{\varphi(a)}\right)}=\lim _{a \downarrow b} \frac{h-g^{\prime}(a)-l_{c} \varphi^{\prime}(a)}{F^{\prime}(a) \varphi(a)+F(a) \varphi^{\prime}(a)-F(c) \varphi^{\prime}(a)} \\
& =\frac{h-g^{\prime}(b)-l_{c} \varphi^{\prime}(b)}{F^{\prime}(b) \varphi(b)+F(b) \varphi^{\prime}(b)-F(c) \varphi^{\prime}(b)}=\frac{k(b)}{\psi^{\prime}(b)-F(c) \varphi^{\prime}(b)},
\end{aligned}
$$


where the second equality follows from an application of L'Hospital's rule. In contrast with equation (2.25) in [1], this implies that the first order smooth fit of the singular control at $b$ can be derived by a limiting argument from the continuous fit of a family of impulse control problems at $b$. Here, the first order smooth fit holds at any $b \in(c, d)$, not only at $b^{*}$, which we will soon discover to be the optimal reflection barrier.

Equations (2.24) and (2.25) imply that $\beta^{\prime}\left(b^{*}\right)=0$ and $\beta^{\prime \prime}\left(b^{*}\right)<0$. Therefore, $b^{*}$ is a local maximum of the function $b \rightarrow \beta(b)$. On the other hand, since we assumed that the uniqueness of the solution to (2.24), $b^{*}$ is the unique local extremum of the function $b \rightarrow \beta(b)$. Let us argue that $b^{*}$ is the global maximum of this function: Assume there exists a point $m \neq b^{*}$ where the maximum of the function $b \rightarrow \beta(b)$ is attained. Then there would exist local minimum $n \in\left(m, b^{*}\right)$ of the function $b \rightarrow \beta(b)$ which contradicts the fact that $b^{*}$ is the unique local extremum of this function. Note that $b \rightarrow \beta(b)=k(b) / \psi^{\prime}(b)$ may not be concave.

Recall the definition of the function $H$ from (2.27). Using (2.15) and (2.21) we can calculate the derivative of $H$ with respect to $b$ as

$$
\begin{aligned}
\left.\frac{\partial}{\partial b} H(x ; b)\right|_{b=b^{*}} & =\left.\frac{-h+g^{\prime}(b)+\varphi^{\prime}(b)\left(\beta(b)(F(b)-F(c))+l_{c}\right)+\left(\beta^{\prime}(b)(F(b)-F(c))+\beta(b) F^{\prime}(b)\right) \varphi(b)}{\varphi\left(F^{-1}(x)\right)}\right|_{b=b^{*}} \\
& =\left.\frac{-h+g^{\prime}(b)+\beta(b) \psi^{\prime}(b)+l_{c} \varphi^{\prime}(b)+\beta^{\prime}(b) F(b) \varphi(b)-F(c)\left(\beta(b) \varphi^{\prime}(b)+\beta^{\prime}(b) \varphi(b)\right)}{\varphi\left(F^{-1}(x)\right)}\right|_{b=b^{*}} \\
& =\left.\frac{\beta^{\prime}(b)(F(b)-F(c)) \varphi(b)}{\varphi\left(F^{-1}(x)\right)}\right|_{b=b^{*}}=0 .
\end{aligned}
$$

Here, the second equality follows from the definition of $F$ in $(2.3)$, and the third equality follows from the definition of $\beta(b)$ in (2.28) and (2.23). Note that,

$$
\frac{\partial}{\partial b} H(x ; b)=0 \quad \text { if and only if } b=b^{*} .
$$

On the other hand,

$$
\left.\frac{\partial^{2}}{\partial b^{2}} H(x ; b)\right|_{b=b^{*}}=\left.\frac{\beta^{\prime \prime}(b)(F(b)-F(c)) \varphi(b)+\beta^{\prime}(b) F^{\prime}(b) \varphi(b)+\beta^{\prime}(b)(F(b)-F(c)) \varphi^{\prime}(b)}{\varphi\left(F^{-1}(x)\right)}\right|_{b=b^{*}}<0
$$

since $\beta^{\prime}\left(b^{*}\right)=0, \beta^{\prime \prime}\left(b^{*}\right)<0$ and $F$ is increasing. Now, (2.27), (2.30), 2.31) and (2.32), together with the fact that $b \rightarrow \beta(b)$ is maximized at $b^{*}$ imply that

$$
W^{b *}(x)=\sup _{b \in(c, d)} W^{b}(x), \quad x \in[F(c), F(d)) .
$$

The proof of our assertion follows since it is immediate from (2.33) that $u^{b^{*}}(x)=\sup _{b \in[c, d)} u^{b}(x)$, for all $x \in$ $[c, d)$.

We can extend our results to solve constrained optimization problems. A dividend payout problem with solvency constraints was recently solved by Paulsen [10]. In this problem, the firm is allowed to pay dividends only if the cash flow process $X$ is greater than certain (pre-determined) value $\tilde{b}$. Here, we provide a simpler proof to this result, using the characterization we provided for the optimal reflection barrier in Proposition 2.1

Corollary 2.2. Assume that the assumptions of Proposition 2.1 hold. Let $\mathfrak{\mathfrak { A }}$ be the set of $Z \in \mathfrak{A}$ such that

$$
Z_{t}-Z_{0}=\int_{(0, t)} 1_{\left\{X_{s}=b\right\}} d Z_{s}, \quad b \leq \tilde{b} \in(c, d)
$$


for a fixed $\tilde{b}$ and define

$$
\hat{b} \triangleq\left\{\begin{array}{lll}
b^{*} & \text { if } \quad b^{*} \leq \tilde{b}, \\
\tilde{b} & \text { if } \quad b^{*}>\tilde{b} .
\end{array}\right.
$$

Let us also define $\hat{Z}$ by replacing $b$ with $\hat{b}$ in (2.34). Then

$$
v(x) \triangleq \sup _{Z \in \tilde{\mathfrak{A}}} J^{Z}(x)=J^{\hat{Z}}(x) .
$$

Proof. The proof follows since under the assumptions of Proposition 2.1 the functions $b \rightarrow \beta(b), b \in[c, d)$, $b \rightarrow H(x ; b), x \in[c, d)$ have a unique maximum at $b^{*}$. (See the proof of Proposition 2.1).

\subsection{The Optimality of Local Time Strategies in the Class of Monotone Increasing Con- trols}

Let us write the value function $v(x)$, explicitly and make some observations on it.

$$
v(x)= \begin{cases}v_{0}(x) \triangleq \varphi(x)\left(\beta^{*}(F(x)-F(c))+l_{c}\right)+g(x), & x \in\left[c, b^{*}\right], \\ h \cdot\left(x-b^{*}\right)+v_{0}\left(b^{*}\right), & x \in\left[b^{*}, d\right)\end{cases}
$$

where the second equation is obtained by

$$
\begin{aligned}
K\left(x, b^{*}\right)+u_{0}^{b^{*}}\left(b^{*}\right)+g(x) & =h \cdot\left(x-b^{*}\right)-g(x)+g\left(b^{*}\right)+\varphi\left(b^{*}\right)\left(\beta^{*}\left(F\left(b^{*}\right)-F(c)\right)+l_{c}\right)+g(x) \\
& =h \cdot\left(x-b^{*}\right)+v_{0}\left(b^{*}\right) .
\end{aligned}
$$

It is worth noting that $v(c)=0$.

Remark 2.1. $\quad$ (a) The first and the second derivative of $v(x)$ on $\left(c, b^{*}\right)$ are

$v^{\prime}(x)=\beta^{*} \psi^{\prime}(x)+\left(l_{c}-\beta^{*} F(c)\right) \varphi^{\prime}(x)+g^{\prime}(x) \quad$ and $\quad v^{\prime \prime}(x)=\beta^{*} \psi^{\prime \prime}(x)+\left(l_{c}-\beta^{*} F(c)\right) \varphi^{\prime \prime}(x)+g^{\prime \prime}(x)$.

Evaluating these expressions at $b^{*}$ we obtain

$$
\begin{aligned}
& \left.v^{\prime}(x)\right|_{x=b^{*}}=\frac{k\left(b^{*}\right)}{\psi^{\prime}\left(b^{*}\right)-F(c) \varphi^{\prime}\left(b^{*}\right)}\left(\psi^{\prime}(x)-F(c) \varphi^{\prime}(x)\right)+h-\left.k(x)\right|_{x=b^{*}}=h, \\
& \left.v^{\prime \prime}(x)\right|_{x=b^{*}}=\frac{k\left(b^{*}\right)}{\psi^{\prime}\left(b^{*}\right)-F(c) \varphi^{\prime}\left(b^{*}\right)}\left(\psi^{\prime \prime}(x)-F(c) \varphi^{\prime \prime}(x)\right)-\left.k^{\prime}(x)\right|_{x=b^{*}}=0 .
\end{aligned}
$$

We used (2.23) and (2.26) to obtain the first expression and (2.24) to obtain the second expression. Note that these smooth fit conditions are the two boundary conditions that are frequently imposed to solve the singular control problems in an ordinary Hamilton-Jacobi-Bellman (HJB) approach. In that approach, after the solution is constructed, the assumptions are verified using a verification lemma. However, the smooth fit conditions need not necessarily hold and the HJB approach is unable to tell the sufficient conditions for the smooth fit to hold. Using our alternative methodology, in Proposition 2.1, we are able to list some sufficient conditions for the value function to be $\mathbf{C}^{2}$ on all of its domain.

Furthermore,

$$
(\mathcal{A}-\alpha) v(x)=(\mathcal{A}-\alpha) g(x)=-f(x) \quad \text { for } \quad x \in\left(c, b^{*}\right] .
$$

(b) Under assumption (iii) of Propostion 2.1 the function $b \rightarrow k(b) /\left(\psi^{\prime}(b)-F(c) \varphi^{\prime}(b)\right)$ is maximized at $b^{*}$. Therefore, using the first equation in (2.38) it can be checked that $v^{\prime}(x) \geq h, x \in(c, d)$. 
(c) Since $b^{*}$ satisfies (2.24), then we have that

$$
\frac{k\left(b^{*}\right)}{\psi^{\prime}\left(b^{*}\right)-F(c) \varphi^{\prime}\left(b^{*}\right)}=\frac{k^{\prime}\left(b^{*}\right)}{\psi^{\prime \prime}\left(b^{*}\right)-F(c) \varphi^{\prime \prime}\left(b^{*}\right)} .
$$

Now using, the second equation in (2.38) we have that

$$
v^{\prime \prime}(x)=\frac{k^{\prime}\left(b^{*}\right)}{\psi^{\prime \prime}\left(b^{*}\right)-F(c) \varphi^{\prime \prime}\left(b^{*}\right)}\left(\psi^{\prime \prime}(x)-F(c) \varphi^{\prime \prime}(x)\right)-k^{\prime}(x), \quad x \in\left(c, b^{*}\right]
$$

If either

$$
\varphi^{\prime \prime}(x)-F(c) \varphi^{\prime \prime}(x)>0, \quad x \in\left(c, b^{*}\right] \quad \text { and } \quad x \rightarrow \frac{k^{\prime}(x)}{\psi^{\prime \prime}(x)-F(c) \varphi^{\prime \prime}(x)} \quad \text { is a decreasing function on }\left(c, b^{*}\right],
$$

or

$\varphi^{\prime \prime}(x)-F(c) \varphi^{\prime \prime}(x)<0, \quad x \in\left(c, b^{*}\right] \quad$ and $\quad x \rightarrow \frac{k^{\prime}(x)}{\psi^{\prime \prime}(x)-F(c) \varphi^{\prime \prime}(x)} \quad$ is an increasing function on $\left(c, b^{*}\right]$,

then $v^{\prime \prime}(x) \leq 0, x \in\left(c, b^{*}\right]$. Note from (2.37) that $v^{\prime \prime}(x)=0$ on $x \in\left[b^{*}, d\right)$.

(d) For $x \in\left[b^{*}, d\right)$

$$
\begin{aligned}
(\mathcal{A}-\alpha) v(x) & =(\mathcal{A}-\alpha)\left(h \cdot\left(x-b^{*}\right)+v_{0}\left(b^{*}\right)\right)=\mu(x) h-\alpha h \cdot\left(x-b^{*}\right)-\alpha v_{0}\left(b^{*}\right), \\
& \leq \mu(x) h-\alpha v_{0}\left(b^{*}\right) \leq \mu\left(b^{*}\right) h-\alpha v_{0}\left(b^{*}\right)=\lim _{x \downarrow b^{*}}(\mathcal{A}-\alpha) v(x) \\
& =\lim _{x \uparrow b^{*}}(\mathcal{A}-\alpha) v(x)=-\lim _{x \uparrow b^{*}} f(x)=-f\left(b^{*}\right) \leq-f(x)
\end{aligned}
$$

if we assume that the maximums of the functions $x \rightarrow \mu(x)$ and $x \rightarrow f(x)$ on the interval $\left[b^{*}, d\right)$ are attained at $b^{*}$ (for e.g. if both $x \rightarrow f(x)$ and $x \rightarrow \mu(x)$ are non-increasing on $\left[b^{*}, d\right)$ ), and that these functions are both continuous at $b^{*}$. Note that the identity $\lim _{x \downarrow b^{*}}(\mathcal{A}-\alpha) v(x)=\lim _{x \uparrow b^{*}}(\mathcal{A}-\alpha) v(x)$ is due to (2.38).

The following proposition gives sufficient conditions under which the local time strategies are optimal in the class of all increasing strategies.

Proposition 2.3. Assume that the assumptions of Proposition 2.1 hold. Consider the process

$$
d X_{t}=\mu\left(X_{t}\right) d t+\sigma\left(X_{t}\right) d W_{t}-d \xi_{t}
$$

in which $\xi_{t}$ is an $\left\{\mathcal{F}_{t}\right\}$-adapted, non-decreasing and right-continuous process (except possibly at zero) such that $\mathbb{E}^{x}\left[\int_{0}^{\infty} e^{-\alpha s} d \xi_{s}\right]<\infty$. We denote the family of such controls by $\mathcal{C}$. Let us assume that $x \rightarrow \sigma(x), x \in(c, d)$ is a bounded function, the maximums of the functions $x \rightarrow \mu(x), x \rightarrow f(x)$ on $x \in\left[b^{*}, d\right)$ is attained st $b^{*}$, and that both $\mu(\cdot)$ and $f(\cdot)$ are continuous at $b^{*}$. We further assume that either (2.42) or (2.43) holds. Then $x \rightarrow v(x)$, $x \in(c, d)$ defined in (2.37) satisfies $v(x) \geq J^{\xi}(x), x \in(c, d)$, for any $\xi \in \mathcal{C}$, in which

$$
J^{\xi}(x)=h \xi_{0}(x)+\mathbb{E}^{x-\xi_{0}(x)}\left[\int_{0}^{\infty} e^{-\alpha s} f\left(X_{s}\right) d s+\int_{(0, \infty)} e^{-\alpha s} h d \xi_{s}\right] .
$$

Proof. We first apply Itô's formula to $e^{-\alpha t} v\left(X_{t}\right)$ and get

$$
\begin{aligned}
e^{-\alpha t} v\left(X_{t}\right) & =v(x)+\int_{0}^{t} e^{-\alpha s}(\mathcal{A}-\alpha) v\left(X_{s}\right) d s-\int_{0}^{t} e^{-\alpha s} v^{\prime}\left(X_{s-}\right) d \xi_{s}+\int_{0}^{t} e^{-\alpha s} \sigma\left(X_{s}\right) v^{\prime}\left(X_{s}\right) d W_{s} \\
& +\sum_{0<s \leq t} e^{-\alpha s}\left(v\left(X_{s}\right)-v\left(X_{s-}\right)+v^{\prime}\left(X_{s-}\right) \Delta X_{s}\right),
\end{aligned}
$$


in which $\Delta X_{s}=X_{s-}-X_{s}, s \geq 0$. From equation 2.46) and Remark2.1(b), (c) and (e), it follows that

$$
\begin{aligned}
v(x) & =\int_{0}^{t} h e^{-\alpha s} d \xi_{s}-\int_{0}^{t} e^{-\alpha s}\left(h-v^{\prime}\left(X_{s-}\right)\right) d \xi_{s}-\int_{0}^{t} e^{-\alpha s}(\mathcal{A}-\alpha) v\left(X_{s}\right) d s \\
& -\int_{0}^{t} \sigma\left(X_{s}\right) e^{-\alpha s} v^{\prime}\left(X_{s}\right) d W_{s}-\sum_{0<s \leq t} e^{-\alpha s}\left(v\left(X_{s}\right)-v\left(X_{s-}\right)+v^{\prime}\left(X_{s-}\right) \Delta X_{s}\right)+e^{-\alpha t} v\left(X_{t}\right) \\
& \geq \int_{0}^{t} h e^{-\alpha s} d \xi_{s}+\int_{0}^{t} e^{-\alpha s} f\left(X_{s}\right) d s-\int_{0}^{t} \sigma\left(X_{s}\right) e^{-\alpha s} v^{\prime}\left(X_{s}\right) d W_{s} \\
& -\sum_{0<s \leq t} e^{-\alpha s}\left(v\left(X_{s}\right)-v\left(X_{s-}\right)+v^{\prime}\left(X_{s-}\right) \Delta X_{s}\right)+e^{-\alpha t} v\left(X_{t}\right) \\
\geq & \int_{0}^{t} h e^{-\alpha s} d \xi_{s}+\int_{0}^{t} e^{-\alpha s} f\left(X_{s}\right) d s-\int_{0}^{t} \sigma\left(X_{s}\right) e^{-\alpha s} v^{\prime}\left(X_{s}\right) d W_{s} \\
& -\sum_{0<s \leq t} e^{-\alpha s}\left(v\left(X_{s}\right)-v\left(X_{s-}\right)+v^{\prime}\left(X_{s-}\right) \Delta X_{s}\right) .
\end{aligned}
$$

The last line follows because $v$ is positive: $v(c)=0$ and $v^{\prime}(x) \geq h \geq 0, x \in(c, d)$, by $\operatorname{Remark} 2.1$ (b).

We have that

$$
\mathbb{E}\left[\int_{0}^{t} e^{-\alpha s} \sigma\left(X_{s}\right) v^{\prime}\left(X_{s}\right) d W_{s}\right]=0
$$

since $v^{\prime}(x)$ and $\sigma(x)$ are bounded. On the other hand, since $v^{\prime \prime}(x) \leq 0$ (see Remark 2.1 (d)) for any $x>y$

$$
v(x)-v(y)-v^{\prime}(x)(x-y)=\int_{y}^{x}\left(v^{\prime}(u)-v^{\prime}(x)\right) d u \geq 0,
$$

which implies that

$$
-\sum_{0<s \leq t} e^{-\alpha s}\left(v\left(X_{s}\right)-v\left(X_{s-}\right)+v^{\prime}\left(X_{s-}\right) \Delta X_{s}\right) \geq 0
$$

Now 2.47, 2.48, 2.49) imply that

$$
v(x) \geq \mathbb{E}^{x}\left[\int_{0}^{t} h \cdot e^{-\alpha s} d \xi_{s}+\int_{0}^{t} e^{-\alpha s} f\left(X_{s}\right) d s\right]
$$

for all $t>0$, which implies that $v(x) \geq J^{\xi}(x)$ for all $x \in(c, d)$ after taking a limit as $t \rightarrow \infty$. The exchange of limit and integration is possible due to Assumption 2.1 and the definition of $\mathcal{C}$ as a result of an application of bounded convergence theorem.

Remark 2.2. We give a useful hint which will be helpful in checking whether $x \rightarrow R(x ; b)+\frac{\varphi(b)}{\varphi\left(F^{-1}(x)\right)} W(F(b)), x \in(F(c), F(d))$ satisfies assumption (ii) of Proposition 2.1 Let us denote

$$
m(x)=\frac{1}{F^{\prime}(x)}\left(\frac{K}{\varphi}\right)^{\prime}(x),
$$

then $R^{\prime}(y ; b)=m(x)$ and $R^{\prime \prime}(y ; b)=m^{\prime}(x) / F^{\prime}(x)$, in which $y \triangleq F(x)$. If $x \rightarrow K(x, b)$ is twice-differentiable at $x \in(c, d)$, then

$$
R^{\prime \prime}(y ; b)[(\mathcal{A}-\alpha) K(x, b)] \geq 0
$$

The inequality is strict if $R^{\prime \prime}(y ; b) \neq 0$. 


\subsection{Examples of Dividend Payment Problems}

Example 2.1. Dividend payout with a Brownian motion with drift (Jeanblanc and Shiryaev [6], Case C): Let us assume that the capital of a company is modeled a Brownian motion with a drift and the managers of the company would like to maximize the amount of dividends payed out. We assume that the company is ruined when the capital becomes 0 (i.e. 0 an absorbing boundary). The right boundary $+\infty$ is natural. The uncontrolled process $X^{0}$ is a Brownian motion with drift

$$
d X_{t}^{0}=\mu d t+\sigma d W_{t}
$$

The value function is defined as

$$
v(x) \triangleq \sup _{\mathfrak{A}}\left\{Z_{0}(x)+\mathbb{E}^{x-Z_{0}(x)}\left[\int_{\left(0, \tau_{0}\right)} e^{-\alpha t} d Z_{t}\right]\right\},
$$

where $\tau_{0}=\inf \left\{t \leq 0 ; X_{t}=0\right\}$.

In this problem, $f(x) \equiv 0$ and $h \equiv 1$ and $K(x, y)=x-y$. As in [6] we take $\sigma=\sqrt{2}$. By solving the equation $(\mathcal{A}-\alpha) v(x)=0$, in which $\mathcal{A}$ is the infinitesimal generator of the uncontrolled process $X^{0}$, we find $\psi(x)=e^{\left(-\frac{\mu}{2}+\Delta\right) x}$ and $\varphi(x)=e^{\left(-\frac{\mu}{2}-\Delta\right) x}$ where $\Delta=\sqrt{\left(\frac{\mu}{2}\right)^{2}+\alpha}$. Hence $F(x)=e^{2 \Delta x}$ and $F^{-1}(x)=\frac{\log x}{2 \Delta}$. Note that $F(0)=1, l_{c}=0$ and $k(x)=1$.

\section{Verification of the Conditions in Proposition 2.1.}

(i) For a given $b>0$, we have that

$$
R(y ; b)=(K / \varphi)\left(F^{-1}(y)\right)=\frac{\log y}{2 \Delta}\left(y^{\frac{\frac{1}{2} \mu+\Delta}{2 \Delta}}-b\right)
$$

on $y>F(0)=1$. This function is differentiable with respect to $y$.

(ii) $R(\cdot ; b)$ is increasing on $y \in[F(0), \infty)$ by $(2.50)$ and $\lim _{y \rightarrow \infty} R(y ; b)=\infty$. We also have that

$$
\frac{1}{\varphi\left(F^{-1}(y)\right)}=\frac{\log y}{2 \Delta}
$$

is increasing on $[F(0), \infty)$ to $\infty$.

On the other hand, $(\mathcal{A}-\alpha) K(x, b)=p(x)$ for every $x>0$, in which $p(x) \triangleq \mu-\alpha(x-b)$. This linear function $p(x)$ has only one positive root at say, $k$. Then by (2.51), $R(y ; b)$ is convex on $y \in[F(0), F(k))$ and concave on $y \in(F(k), \infty)$. Observe from (2.53) that $1 / \varphi\left(F^{-} 1(y)\right)$ is concave.

(iii) From (2.24), Since $k^{\prime}(x)=0$ for $x \in[0, \infty)$, Equations (2.24) and (2.25) become

$$
\begin{gathered}
\psi^{\prime \prime}(b)=F(0) \varphi^{\prime \prime}(b), \\
\psi^{\prime \prime \prime}\left(b^{*}\right)-\varphi^{\prime \prime \prime}\left(b^{*}\right) F(0)>0 .
\end{gathered}
$$

Since $\psi^{\prime \prime}(\cdot)$ is increasing and $\varphi^{\prime \prime}(\cdot)$ is decreasing on $[0, \infty)$, equation $(2.55)$ holds for all $x \in[0, \infty)$. Moreover, since $\psi^{\prime \prime}(0)<\varphi^{\prime \prime}(0)$ and $0=\lim _{x \rightarrow \infty} \varphi^{\prime \prime}(x)<\lim _{x \rightarrow \infty} \psi^{\prime \prime}(x)=\infty$, there exists a unique solution to (2.54).

Verification of the Conditions in Proposition 2.3; The only non-trivial condition to check is whether $v^{\prime \prime}(x) \leq 0$ for $x \in(0, \infty)$. It can be shown that $\psi^{\prime \prime}(x)-F(0) \varphi^{\prime \prime}(x)<0$ on $x \in\left(0, b^{*}\right)$, by the same argument that we used to prove the uniqueness of the root of (2.54) and the concavity of $v$ follows from Remark 2.1-c. Hence we conclude that the local time strategy at $b^{*}$ is optimal among all the admissible strategies. 
Now, $v_{0}(\cdot)$, defined in 2.37) can be computed as

$$
\begin{aligned}
v(x) & =\varphi(x) W^{b^{*}}(F(x))=\beta^{*}(F(x)-1) \varphi(x)=\beta^{*}\left(e^{2 x \Delta}-1\right) e^{-(\mu / 2+\Delta) x} \\
& =\beta^{*} e^{-\mu x / 2}\left(e^{\Delta x}-e^{-\Delta x}\right)=2 \beta^{*} e^{-\mu x / 2} \sinh (x \Delta) .
\end{aligned}
$$

The solution to this problem is then

$$
v(x)= \begin{cases}v_{0}(x), & 0 \leq x \leq b^{*}, \\ v_{0}\left(b^{*}\right)+x-b^{*}, & b^{*} \leq x,\end{cases}
$$

which coincides with the solution that is computed by Jeanblanc and Shiryaev [6] by using the ordinary HJB approach, which is specific to the modeling assumptions. Figure 1 shows the value function after applying the transformation (2.19), the slope function $b \rightarrow \beta(b), b \in(c, d)$, the value function and its derivative when the parameters are $(\mu, \alpha)=(0.15,0.2)$. The optimal reflection point is $b^{*}=0.736246$ and $\beta\left(b^{*}\right)=1.16523$.

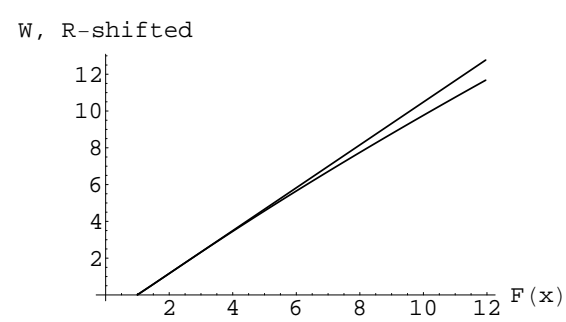

(a)

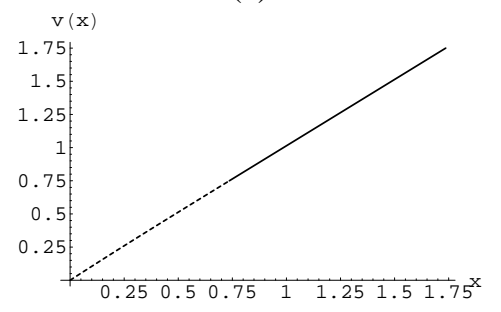

(c)

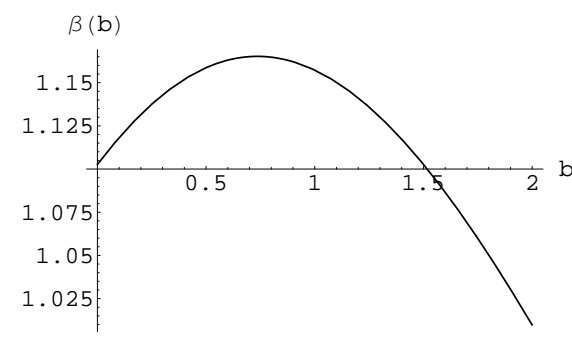

(b)

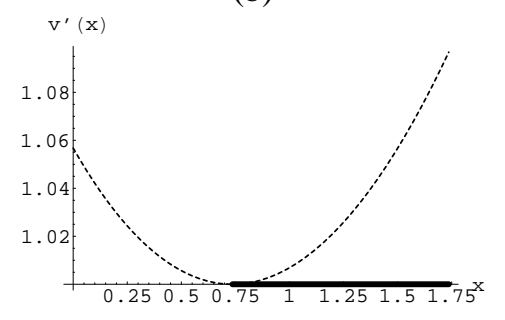

(d)

Figure 1: The analysis of the singular control problem of Jeanblanc and Shiryaev [6] with parameters $(\mu, \alpha)=(0.15,0.2)$ : (a) The function $x \rightarrow R\left(F(x), b^{*}\right)+\varphi\left(b^{*}\right) / \varphi(x), x \in(c, d)$ and its linear majorant. (b) The graph of $b \rightarrow \beta(b)$ (see (2.28). It attains its maximum at $b^{*}$. (c) The value function $v(x)$ with $b^{*}=0.736246$ and $\beta^{*}=1.16523$. (d) The derivative $v^{\prime}(x)$ : It is $v_{0}^{\prime}(x)$ on $0 \leq x \leq b^{*}$ and $v^{\prime}(x)=1$ on $b^{*} \leq x$ (lower line). The derivative $v_{0}^{\prime}(x)$ on $b^{*} \leq x$ is also shown to illustrate that $v_{0}^{\prime \prime}\left(b^{*}\right)=0$.

Example 2.2. Dividend payout with a square root process: We solve the problem defined in (2.52) when the cash flow of the company is modeled by the following square root process:

$$
d X_{t}^{0}=\left(1-2 \rho X_{t}^{0}\right) d t+2 \sqrt{X_{t}^{0}} d W_{t}, \quad X_{0}^{0}=x>0 .
$$

The solutions of $(\mathcal{A}-\alpha) v(x)=0$ are

$$
\psi(x)=x^{-1 / 4} \exp \left(\frac{\rho x}{2}\right) M_{-\frac{\alpha}{2 \rho}+\frac{1}{4},-\frac{1}{4}}(\rho x), \quad \varphi(x)=x^{-1 / 4} \exp \left(\frac{\rho x}{2}\right) W_{-\frac{\alpha}{2 \rho}+\frac{1}{4},-\frac{1}{4}}(\rho x),
$$

in which $W_{-\frac{\alpha}{2 \rho}+\frac{1}{4},-\frac{1}{4}}$ and $M_{-\frac{\alpha}{2 \rho}+\frac{1}{4},-\frac{1}{4}}$ are Whittaker functions. (See Appendix 1.26 of Borodin and Salminen 
[2] as well as Chapter 2.1.11.) The Whittaker functions are defined as

$$
\begin{aligned}
& W_{-\frac{\alpha}{2 \rho}+\frac{1}{4},-\frac{1}{4}}\left(\frac{x^{2}}{2}\right)=2^{\frac{\alpha}{2 \rho}-\frac{1}{4}} \sqrt{x} D_{-\alpha / \rho}(x), \quad x \geq 0, \\
& M_{-\frac{\alpha}{2 \rho}+\frac{1}{4},-\frac{1}{4}}\left(\frac{x^{2}}{2}\right)=\frac{\Gamma((1+\alpha / \rho) / 2)}{2 \sqrt{\pi}} \sqrt{x}\left(D_{-\alpha / \rho}(-x)-D_{-\alpha / \rho}(x)\right), \quad x \geq 0,
\end{aligned}
$$

in which $\Gamma$ stands for the Gamma function $\Gamma(x)=\int_{0}^{\infty} u^{x-1} e^{-u} d u$ and $D_{\nu}(\cdot)$ is the parabolic cylinder function, which is defined as

$$
D_{\nu}(x) \triangleq 2^{\nu / 2} e^{-x^{2} / 4} H_{v}\left(\frac{x}{\sqrt{2}}\right), \quad x \in \mathbb{R},
$$

in terms of the Hermite polynomial, $H_{\nu}$, of order $\nu$, whose integral representation is given by

$$
\mathcal{H}_{\nu}(z)=\frac{1}{\Gamma(-\nu)} \int_{0}^{\infty} e^{-t^{2}-2 t z} t^{-\nu-1} d t, \quad \operatorname{Re}(\nu)<0 .
$$

See e.g. Lebedev [8]. The Hermite polynomials satisfy $\mathcal{H}_{\nu}^{\prime}(z)=2 \nu \mathcal{H}_{\nu-1}(z), z \in \mathbb{R}$.

\section{Verification of the Conditions in Proposition 2.1;}

(i) Note that

$$
R(F(x) ; b)=\frac{K(x, b)}{\varphi(x)}=\frac{x-b}{\varphi(x)}
$$

and the differentiability of $R(y ; b)$ comes from that of $F(\cdot)$ and $\varphi(\cdot)$.

(ii) Since

$$
\frac{\partial}{\partial x} \frac{K(x, b)}{\varphi(x)}=\frac{\varphi(x)-(x-b) \varphi^{\prime}(x)}{\varphi(x)^{2}} \geq 0
$$

for $x \geq b$, using $(2.50)$, it can be seen that $R(y ; b)$ is increasing on $y \in(F(b), \infty)$ to $\infty$.

On the other hand, $(\mathcal{A}-\alpha)(x-b)=p(x)$ for every $x>0$, in which $p(x)=(1-2 \rho x)-\alpha(x-b)$. This linear function $p(x)$ has one positive root at say, $k$. Then $R(y ; \cdot)$ is convex on $y \in[F(0), F(k))$ and concave on $y \in(F(k), \infty)$.

The facts that $1 / \varphi\left(F^{-1}(y)\right)$ is increasing to $\infty$ and concave on $[F(0), \infty)$ can be similarly shown (by replacing $K(x, b)$ in (2.60) with unity).

(iii) We need to verify that (2.54) and (2.55) hold. In fact, we will see that when $\alpha>0$, the unique solution of (2.54) or the optimal reflection level satisfies $b^{*} \in(0,1 /(2 \rho))$. This result is very intuitive, since $x=1 /(2 \rho)$ is the mean-reversion level of $X$.

The functions $\psi(\cdot)$ and $\varphi(\cdot)$ both solve the differential equation

$$
(1-2 \rho x) w^{\prime}(x)+2 x f w^{\prime \prime}(x)-\alpha w(x)=0, \quad x \in(0, \infty) .
$$

Also, we know from their representation in (2.2) that

$$
\psi(x)>0, \psi^{\prime}(x)>0 ; \quad \varphi(x)>0, \varphi^{\prime}(x)<0, \quad x \in(0, \infty) .
$$

Evaluating (2.62) at $x=1 /(2 \rho)$ for $w=\psi$ and $w=\varphi$, we obtain

$$
2 x \psi^{\prime \prime}\left(\frac{1}{2 \rho}\right)=\alpha \psi\left(\frac{1}{2 \rho}\right) \quad \text { and } 2 x \psi^{\prime \prime}\left(\frac{1}{2 \rho}\right)=\alpha \psi\left(\frac{1}{2 \rho}\right),
$$


from which it follows that, for $\alpha>0$,

$$
\frac{\psi^{\prime \prime}\left(\frac{1}{2 \rho}\right)}{\varphi^{\prime \prime}\left(\frac{1}{2 \rho}\right)}=\frac{\psi\left(\frac{1}{2 \rho}\right)}{\varphi\left(\frac{1}{2 \rho}\right)}=F\left(\frac{1}{2 \rho}\right) \geq F(0)>0,
$$

where the first inequality follows from the fact that $F$ defined in $(2.3)$ is increasing. If $\alpha=0$, then $\psi^{\prime \prime}(1 /(2 \rho))=$ $\varphi^{\prime \prime}(1 /(2 \rho))=0$.

First, we will show that (2.54) has a unique solution in $(1,1 /(2 \rho)]$, and show that this solution indeed satisfies (2.55). Next, we will show that the same equation does not have a solution in $(1 /(2 \rho), \infty)$. To establish our first goal, let us collect some information on the behavior of the functions $\varphi(\cdot)$ and $\psi(\cdot)$ over this interval. From (2.62) with $f=\varphi$, we see that when

$$
\varphi^{\prime \prime}(x)>0, \quad x \in\left(0, \frac{1}{2 \rho}\right],
$$

since $\varphi^{\prime}(x)<0$ and $\varphi(x)>0$ over the same interval. Differentiating 2.3 we obtain

$$
(2 \rho+\alpha) w^{\prime}(x)-(3-2 \rho x) w^{\prime \prime}(x)=2 x w^{\prime \prime \prime}(x), \quad x \in(0, \infty),
$$

for $w=\psi$ or $w=\varphi$. Using (2.67) it can be seen that

$$
\varphi^{\prime \prime \prime}(x)<0, \quad x \in\left(0, \frac{1}{2 \rho}\right],
$$

using the fact that $\varphi^{\prime}(x)<0$ and $\varphi^{\prime \prime}(x)>0$ on the same interval.

After simplifying the expression for $\psi(\cdot)$ in 2.56 , we write

$$
\psi(x)=H_{-\alpha / \rho}(-\sqrt{\rho x})-H_{-\alpha / \rho}(\sqrt{\rho x}) .
$$

The second derivative of $\psi(\cdot)$, then can be computed as

$\psi^{\prime \prime}(x)=-\frac{\alpha}{4 \sqrt{\rho}} x^{-3 / 2}\left(H_{-\alpha / \rho-1}(-\sqrt{\rho x})+H_{-\alpha / \rho-1}(\sqrt{\rho x})\right)+\frac{\alpha}{4}\left(\frac{\alpha}{\rho}+1\right) x^{-1}\left(H_{-\alpha / \rho-2}(-\sqrt{\rho x})+H_{-\alpha / \rho-2}(\sqrt{\rho x})\right)$

from which it follows that

$$
\lim _{x \rightarrow 0+} \psi^{\prime \prime}(x)=-\infty .
$$

With the help of 2.67 with $w=\psi$, observe that

$$
\text { if } x \in(0,1 /(2 \rho)) \text {, then } \psi^{\prime \prime \prime}(x)>0 \text {, if } \psi^{\prime \prime}(x)<0
$$

since $\psi^{\prime}(x)>0$. It follows from 2.65) and (2.66) that $\psi^{\prime \prime}(1 /(2 \rho))>0$. Now, this fact together with (2.71) imply that

$$
\psi^{\prime \prime}(x) \leq 0 \quad \text { for } x \in\left(0, x_{0}\right], \quad \text { where } x_{0} \in\left(0, \frac{1}{2 \rho}\right) .
$$

And it also follows from 2.72 that

$$
\psi^{\prime \prime}(x)>0, \quad x \in\left(x_{0}, \frac{1}{2 \rho}\right) .
$$

At this point, we can state that there exists a solution, $b^{*} \in\left(x_{0}, 1 /(2 \rho)\right)$ to (2.54) as a result of (2.65), 2.66), (2.73) and the intermediate value theorem.

Let us prove that at $b^{*}, \psi^{\prime \prime \prime}\left(b^{*}\right) \geq 0$. If $\psi^{\prime \prime \prime}\left(b^{*}\right)<0$, then there would exist a point $\tilde{x}_{0} \in\left(x_{0}, b^{*}\right)$ such that

$$
\psi^{\prime \prime \prime}\left(\tilde{x}_{0}\right)=0, \quad \psi^{(4)}\left(\tilde{x}_{0}\right)<0
$$


in which $\psi^{(4)}$ stands for the fourth derivative of $\psi$. Differentiating (2.67) we write

$$
(4 \rho+\alpha) w^{\prime \prime}(x)-(5-2 \rho x) w^{\prime \prime \prime}(x)=2 x w^{(4)}(x), \quad x \in(0, \infty) .
$$

Evaluating the left-hand-side of (2.76) (when $w=\psi$ ) at $\tilde{x}_{0}$ we obtain a positive quantity using (2.74) and the equality in (2.75), whereas the right-hand-side of (2.75) due to the inequality in (2.75), which yields a contradiction. Since $\psi^{\prime \prime \prime}\left(b^{*}\right) \geq 0$, and $\varphi^{\prime \prime \prime}\left(b^{*}\right)<0$ by (2.68), $b^{*}$ satisfies (2.55).

Let us show that $b^{*}$ is the only solution to $(2.54)$ in $(0,1 /(2 \rho))$. Assume there exists another solution to (2.54) in $(0,1 /(2 \rho))$, then necessarily there would be at least one more solution to 2.54$)$ in $(0,1 /(2 \rho))$ (This follows from (2.65), (2.66), 2.68), and (2.73). Let us denote the largest three of all of the solutions by $x_{1}<x_{2}<x_{3}$. It can be easily under this assumption

$$
\psi^{\prime \prime}(x)>\varphi^{\prime \prime}(x), \quad x \in\left(x_{1}, x_{2}\right), \quad \text { and } \quad \psi^{\prime \prime \prime}\left(x_{2}\right)<0 .
$$

But this contradicts the fact we have proved above: since $x_{2}$ is a solution to $2.54, \psi^{\prime \prime \prime}\left(x_{2}\right) \geq 0$.

It remains to show that (2.54) does not have a solution in $[1 /(2 \rho), \infty)$. It is clear from $(2.65)$ that $x=1 /(2 \rho)$ is not a solution of (2.54). Let us assume that for $x>1 /(2 \rho), \psi^{\prime \prime}(x)=F(0) \varphi^{\prime \prime}(x)$. Then,

$$
-(1-2 \rho x) \psi^{\prime}(x)+\alpha \psi(x)=-F(0)(1-2 \rho x) \psi^{\prime}(x)+F(0) \alpha \varphi(x),
$$

which implies that

$$
\alpha(F(x)-F(0))=(1-2 \rho x)\left(\frac{\psi^{\prime}(x)-F(0) \varphi^{\prime}(x)}{\varphi(x)}\right) .
$$

Note that the left-hand-side of (2.79) is non-negative because $F$ is increasing. On the other hand the right-hand-side of (2.79) is negative. This yields a contradiction.

Verification of the Conditions in Proposition 2.3: The only non-trivial condition to check is whether $v^{\prime \prime}(x) \leq 0$ for $x \in(0, \infty)$. It is clear from our analysis above that $\psi^{\prime \prime}(x)-F(0) \varphi^{\prime \prime}(x)<0$ on $x \in\left(0, b^{*}\right)$. The concavity of $v$ follows from Remark 2.1. c.

We can determine $\beta^{*}$ from $(2.26)$ and write down the value function as

$$
v(x)= \begin{cases}v_{0}(x) \triangleq \beta^{*}(\psi(x)-F(0) \varphi(x)), & 0 \leq x \leq b^{*}, \\ v_{0}\left(b^{*}\right)+x-b^{*}, & b^{*} \leq x .\end{cases}
$$

in which $\psi(x)$ and $\varphi(x)$ are given by (2.57) with (2.58). Figure 2 illustrates the function $b \rightarrow \beta(b)$, the value function, $v$, and its derivatives for a special choice of parameters.

\section{Appendix}

Lemma 3.1. Let us assume that assumption (2.8) holds. Then for any stopping time $\tau$ of the filtration $\left(\mathcal{F}_{t}\right)_{t \geq 0}$

$$
\mathbb{E}^{x}\left[\int_{0}^{\tau} e^{-\alpha s} f\left(X_{s}^{0}\right) d s\right]=g(x)-\mathbb{E}^{x}\left[e^{-\alpha \tau} g\left(X_{\tau}^{0}\right)\right],
$$

in which $g$ is defined in (2.9).

Proof. The proof immediately follows from the strong Markov property of the process $X^{0}$. 


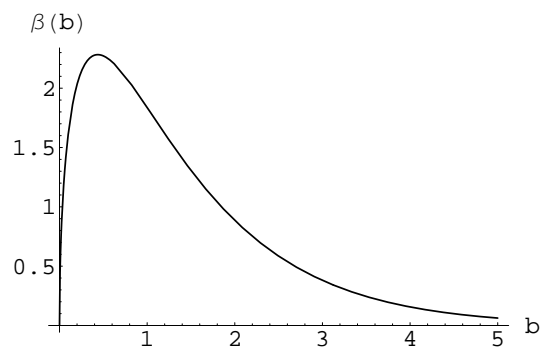

(a)

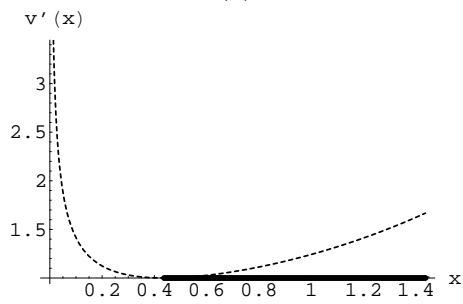

(c)

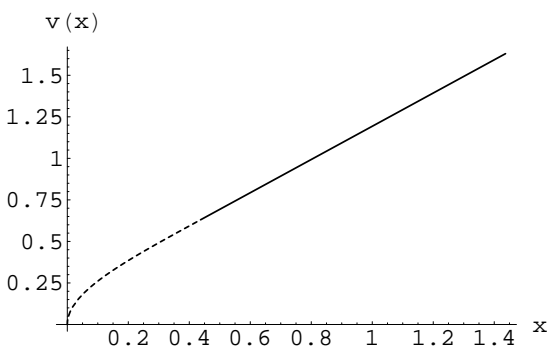

(b)

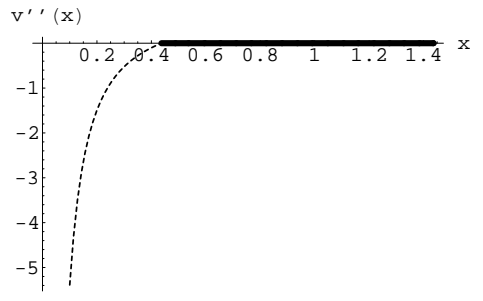

(d)

Figure 2: The dividend payout problem with a square root process with parameters $(\rho, \alpha)=(1,0.2)$ : (a) The graph of $b \rightarrow \beta(b)$ (see (2.28)). It attains its maximum at $b^{*}$. (b) The value function $v(x) . b^{*}=0.4370$ and $\beta^{*}=2.2826$. (c) The derivative $v^{\prime}(x)$ : It is $v_{0}^{\prime}(x)$ on $0 \leq x \leq b^{*}$ and $v^{\prime}(x)=1$ on $b^{*} \leq x$. The derivative $v_{0}^{\prime}(x)$ on $b^{*} \leq x$ is also shown to illustrate that $v_{0}^{\prime \prime}\left(b^{*}\right)=0$.

Lemma 3.2. For any pair $(l, r) \in(c, d)^{2}$, let us define

$$
v_{r}(x) \triangleq \mathbb{E}^{x}\left[e^{-\alpha \tau_{r}} 1_{\left\{\tau_{r}<\tau_{l}\right\}}\right], \quad \text { and } \quad v_{l}(x) \triangleq \mathbb{E}^{x}\left[e^{-\alpha \tau_{r}} 1_{\left\{\tau_{l}<\tau_{r}\right\}}\right], \quad x \in[l, r]
$$

Then

$$
v_{r}(x)=\frac{\psi(l) \varphi(x)-\psi(x) \varphi(l)}{\psi(l) \varphi(r)-\psi(r) \varphi(l)}, \quad \text { and } \quad v_{l}(x)=\frac{\psi(x) \varphi(r)-\psi(r) \varphi(x)}{\psi(l) \varphi(r)-\psi(r) \varphi(l)}, \quad x \in[l, r]
$$

Proof. Both $x \rightarrow v_{r}(x)$ and $x \rightarrow v_{l}(x), x \in(c, d)$ are solutions to $(\mathcal{A}-\alpha) u=0$ with boundary conditions $v_{l}(l)=$ $v_{r}(r)=1$ and $v_{l}(r)=v_{r}(l)=0$. Therefore, we can write them as linear combinations of the homogeneous solutions of $(\mathcal{A}-\alpha) u=0$, and we get $(3.3)$.

\section{References}

[1] E. Bayraktar and M. Egami. The effects of implementation delay on decision-making under uncertainty. Stochastic Processes and Their Applications, 117 (3):333-358, 2007.

[2] A. N. Borodin and P. Salminen. Handbook of Brownian Motion Facts and Formulae. Birkhäuser, Boston, 2002.

[3] S. Dayanik and I. Karatzas. On the optimal stopping problem for one-dimensional diffusions. Stochastic Processes and their Applications, 107 (2):173-212, 2003.

[4] M. Egami. A direct solution method for stochastic impulse control problems of one-dimensional diffusions. Preprint, www.umich.edu/ egami, 2006. 
[5] K. Itô and H. P. McKean. Diffusion processes and their sample paths. Springer-Verlag, New York, 1974.

[6] M. Jeanblanc-Picqué and A. N. Shiryaev. Optimization of the flow of dividends. Russian Math. Surveys, 50 (2):257-277, 1995.

[7] I. Karatzas and S. E. Shreve. Connections between optimal stopping and singular stochastic control i. monotone follower problems. SIAM J. Control Optim., 22 (6):856-877, 1984.

[8] N. N. Lebedev. Special Functions and Their Applications. Dover Publications, New York, 1972.

[9] B. Øksendal and A. Sulem. Applied stochastic controll of jump diffusions. Springer-Verlag, New York, 2005.

[10] J. Paulsen. Optimal dividend payouts for diffusions with solvency constraints. Finance and Stochastics, 7:457-473, 2003. 